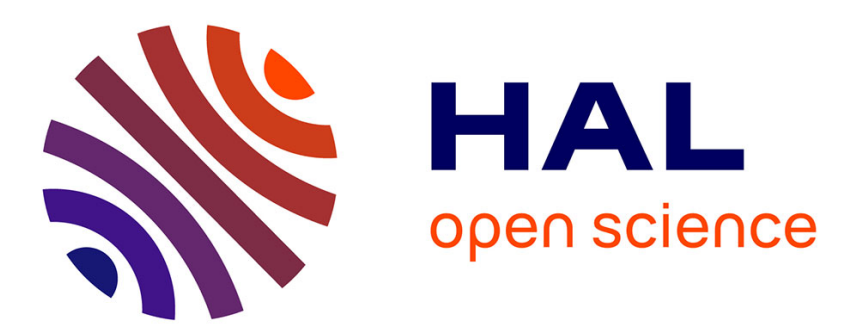

\title{
Sic itur ad astra: quand le janséniste d'Aguesseau aborde le Politique
}

\author{
Isabelle Storez-Brancourt
}

\section{To cite this version:}

Isabelle Storez-Brancourt. Sic itur ad astra: quand le janséniste d'Aguesseau aborde le Politique. 2004. halshs-00188401

\section{HAL Id: halshs-00188401 https://shs.hal.science/halshs-00188401}

Preprint submitted on 16 Nov 2007

HAL is a multi-disciplinary open access archive for the deposit and dissemination of scientific research documents, whether they are published or not. The documents may come from teaching and research institutions in France or abroad, or from public or private research centers.
L'archive ouverte pluridisciplinaire HAL, est destinée au dépôt et à la diffusion de documents scientifiques de niveau recherche, publiés ou non, émanant des établissements d'enseignement et de recherche français ou étrangers, des laboratoires publics ou privés. 
Isabelle STOREZ-BRANCOURT

CR1 - Institut d'Histoire du Droit- UMR 7184

\section{Sic itur ad astra : Quand le janséniste d'Aguesseau aborde le Politique}

«Sic itur ad astra $»^{1}$ ! La question d'aujourd'hui est la suivante : comment s'élève-t-on au firmament de la Politique lorsqu'on s'appelle Henri François d'Aguesseau et que l'on n'est, en ce début du XVIII ${ }^{\mathrm{e}}$ siècle, ni le fils ni le neveu d'un Colbert, ni parent d'un Louvois ou d'un Phélypeaux ? Le jansénisme dont, en ce temps, l'on taxait volontiers les d'Aguesseau, comme bon nombre de familles parlementaires ou anciennement frondeuses, n'apparait pas, au premier regard, comme un tremplin de choix. Pourtant la promotion de d'Aguesseau fut aussi spectaculaire qu'applaudie, aussi agitée en ces débuts que protégée jusqu'en sa fin.

Le chancelier Henri François d'Aguesseau accéda à la Chancellerie de France, le 2 février 1717, tandis que son prédécesseur, Daniel Voysin, venait de mourir la nuit précédente : "L'apoplexie de M. le chancelier Voisin », écrit Dangeau, «fut si violente qu'il mourut une heure après minuit sans que la connoissance lui fût revenue un seul moment. A huit heures du matin, MM. ... rapportèrent les sceaux à $M$. le duc d'Orléans, qui envoya quérir dans le moment $M$. Daguesseau, procureur général, à qui il donna la charge de chancelier et les sceaux $»^{2}$. Rapidité, facilité, telles sont, en résumé, les caractéristiques de la nomination de d'Aguesseau à la chancellerie. Le propos de cette communication repose sur un constat fondamental: celui du déséquilibre impressionnant, dans le cas de d'Aguesseau, entre l'extrême simplicité de son accession à ce premier office de la Couronne ${ }^{3}$, d'une part, et les rapides et très graves difficultés qui ont entravé l'exercice de sa fonction, dès 1718. Le même contraste se remarque d'ailleurs entre le caractère

\footnotetext{
${ }^{1}$ Les amateurs de vieille littérature enfantine se souviendront de ces félicitations faites par son directeur d'école au jeune et futur Savant Cosinus qui vient de recevoir le prix d'excellence...

${ }^{2}$ Marquis de Dangeau, Journal, éd. Soulié, Paris, 1854-1860, t. XVII, p. 14.

${ }^{3}$ Depuis l'abolition de la charge de connétable de France, en 1627, l'office de chancelier de France a, de fait sinon de droit, la dignité de premier grand officier de la Couronne.
} 
largement négatif des jugements portés par les contemporains sur «l'homme politique » qu'était supposé être d'Aguesseau, et la profondeur des marques qu'il a imprimées dans l'Histoire. L'explication de ces contrastes se trouveraitelle dans le caractère et les idées du personnage ou bien dans les circonstances de ce 2 février 1717 ?

Je tenterai d'éclaircir ces paradoxes à la lumière des conditions politiques de la nomination de d'Aguesseau en évoquant différents types de logiques : celle de sa personnalité propre et de sa vision du Politique, d'abord, celle de sa carrière antérieure ou d'un supposé «parti » qui aurait appuyé sa candidature, ensuite. Cela me permettra de mettre en évidence les forces et les faiblesses du choix opéré, en ce 2 février 1717, par le Régent, tant du point de vue du gouvernement royal que du point de vue de d'Aguesseau lui-même.

\section{I - « Le sujet seroit riche pour les nouvellistes, ou même pour les politiques, et je ne suis guère plus l'un que l'autre ${ }^{4}$.}

Par cet aveu à son fils, Henri François d'Aguesseau énonce ce qui lui paraît une évidence : son dégoût de la politique, plus précisément son inaptitude à juger des faits politiques et sociaux de son temps, à analyser le gouvernement et son action. Il se déclare incompétent dans ce domaine qui recouvre aujourd'hui, par excellence, ce que nous appelons la «politique ». En ce sens, le mot même est d'ailleurs fort rare sous la plume de d'Aguesseau, au demeurant presque toujours péjoratif. Prudence ou désintérêt? Difficile de le dire. A s'en tenir aux allusions qu'il consent, dans sa correspondance en particulier, on observe que la politique lui paraît un univers bizarre, certainement dangereux, plein d'obscurité et de traquenards. Dans ses lettres privées, il parle volontiers de «ce pays-ci $»^{5}$, pour désigner le gouvernement ou la cour, un pays où la « servitude " est de bon aloi. Une autre fois, il prête à son père la volonté, en l'introduisant dans la carrière de la magistrature des cours, de le «faire naviguer sur une mer moins tumultueuse que celle du Conseil $»^{7}$. Il se plaint aussi « du tumulte de Paris, et qui pis est, de la Cour $»^{8}$.

\footnotetext{
${ }^{4}$ Lettres inédites du chancelier d'Aguesseau, éd. D.B. Rives, Paris, 1823, 2 vol., in- $8^{\circ}$, t. II, p. 8.

${ }^{5}$ Ibidem, t. II, p. 277.

${ }^{6}$ D'Aguesseau, Discours sur le vie et la mort de M. d'Aguesseau, conseiller d'Etat, éd. 1812, in-12, p. 134.

${ }^{7}$ Ibidem.
} 
Reprenant la même image, il évoque ailleurs «le tourbillon du monde et des affaires $»^{9}$. Une fois même, parlant de sa mère, qui n' aurait pas été, prétend-il, à l'abri de cette tentation, il désigne par le terme de politique l'intrigue et la recherche d'influence : aussi dénonce-t-il «cette espèce de politique dont les femmes d'un génie supérieur sont encore plus touchées que les hommes ${ }^{10}$. Lorsque s'entrebâille la porte du politique, le mot d'ordre paraît silence et secret : "C'est matière de conversation plutôt que de lettre », écrit-il à son fils aîné aux alentours de 1725. D'Aguesseau se jugeait mauvais courtisan, et s'en flattait. En 1726, il supplie donc en termes définitifs : qu'on lui épargne les «almanachs »! qu'on lui fasse grâce « de ce que vous appelez une gazette. Le sujet seroit riche pour les nouvellistes, ou même pour les politiques, et je ne suis guère plus l'un que l'autre »!

Pour être tout à fait honnête, on observera cependant, outre le brin de coquetterie qui affecte certains de ces propos, que la quasi totalité de ces remarques sont concomitantes ou postérieures aux exils qui ont entravé la carrière du ministre. L'amertume n'est pas exclue. En fait, il serait tout à fait abusif d'en rester à une signification strictement pragmatique de la politique : d'Aguesseau, lorsqu'il est porté au ministère en 1717, y arrive avec des conceptions fortes et précises de philosophie politique : on les trouve, éparses, dans ses Mercuriales ${ }^{11}$ et dans ses Instructions sur les études propres à former un Magistrat; bientôt rassemblées, mais inachevées dans son Essai d'une institution au Droit public et, un peu plus tard, dans ses Méditations métaphysiques sur les vraies et fausses idées de la Justice. Pour s'en tenir aux premiers écrits, qui sont regroupés dans le volume I de l'édition in- $4^{\circ}$ de 1759 , j'ai constaté, que si le mot de «politique » n'y apparaissait qu'à peine quinze fois en plus de 550 pages, la préoccupation politique est en fait dominante dans cette partie de l'œuvre du magistrat. Totalement absent des Mercuriales, le mot politique recouvre chez d'Aguesseau des acceptions bien précises : il parle de «corps politique» (sept fois), de «mauvais politiques» (p. 525) ou de « Politique moderne » (p. 287), les uns et les autres assimilés aux «philosophes

\footnotetext{
${ }^{8}$ D'Aguesseau, Euvres complètes, éd. Pardessus, Paris, 1819, 16 vol., t. XVI, Lettres sur divers sujets (correspondance privée, souvent adressée à Valincour), p. 294.

9 D'Aguesseau, Instructions sur les étude propres à former un Magistrat, Euvres du chancelier d'Aguesseau, éd. 1759, in-4 $4^{\circ}, 13$ vol., t. I, p. 286.

${ }^{10}$ Discours sur la vie et la mort de M. d'Aguesseau, conseiller d'Etat, op. cit., p. 22-23.

${ }^{11}$ Discours prononcé par l'un des membres du Parquet devant l'assemblée des chambres des parlements le mercredi qui suivait l'ouverture de la session judiciaire, après la Saint-Martin et Pâques.
} 
politiques » (p. 270); il mentionne aussi «l'ordre politique» (p. 330) comme «la Politique », tout simplement, en quatre occasions, au sens précis de «science de l'organisation de la Cité » 12 , de «Gouvernement Temporel» (p. 542) opposé au Spirituel, qui peut être, dès lors «une fausse Politique » (p. 526), une conception que d'Aguesseau n'hésite pas à déclarer erronée, car il croit à l'existence du «vrai » et du «faux », en philosophie politique comme en religion. Son vocabulaire est celui, classique, de la philosophie politique: gouvernement, République, au sens de «chose publique », plus rarement l'Etat, le service de la Patrie forment le fond de sa réflexion; il raisonne ainsi du Citoyen, du Magistrat, du Souverain... Si «l'homme politique » n'existe pas dans le lexique de d'Aguesseau, pas plus d'ailleurs que dans le vocabulaire de ses contemporains, un terme s'en approchant de façon évidente par la signification que lui accorde l'auteur, est au contraire au cœur de son propos : c'est celui «d'homme public » ${ }^{13}$; de même la rareté de l'emploi de «politique » ne doit pas dissimuler l'intérêt essentiel de d'Aguesseau pour la chose «publique» comme pour le «Droit public»14. Le «Public ${ }^{15}$, qu'il désigne sous la plume de d'Aguesseau «l'opinion publique », ou le «ministère public », parfois même l'Etat, est l'objet central de sa réflexion. Dès lors il n'est plus question d'escamoter l'attrait de d'Aguesseau pour le Politique et ce fait même pose le délicat problème de son « jansénisme politique ».

Une phrase des Mercuriales ouvre des perspectives intéressantes : « Nous avouons, il est vrai », écrit-il, « et nous voudrions pouvoir le dissimuler, que le service public devient tous les jours plus difficile, mais ne croyons pas qu'il puisse jamais devenir impossible à l'homme de bien... ${ }^{16}$ On trouve ici les deux versants opposés de la pensée de d'Aguesseau : d'un côté, il exprime un

\footnotetext{
${ }^{12}$ Une fois, il met ainsi la « Politique » en parallèle avec la « Théologie » (p. 296).

13 D'Aguesseau lui consacre sa VIIIe Mercuriale : L'homme public ou l'attachement du Magistrat au service public. Pâques 1706, tome I, p. 118 et suivantes.

${ }^{14}$ On peut relever, par exemple, outre « Droit public » et « homme public », l'expression « bien public» (t. I, p. 123, p. 209), pour désigner le «bien commun», plus fréquent avant d'Aguesseau et dont on ne trouve qu'une seule mention dans le premier volume (dans la XIX Mercuriale : L'amour de la Patrie) ; on trouve plus fréquemment encore le «service public » (p. 118, 123) ou le « service du Public» (p. 33), ou encore «l'intérêt public» (au singulier ou au pluriel, p. 123). On trouve aussi «bien public» (p. 123 et 209), enfin « ordre public» (p. 311, 330).

${ }^{15}$ Entre autres exemples, p. 135, 140, 146, 147...

16 VIIIe Mercuriale. L'homme public ou l'attachement du Magistrat au service public, t. I, p. 123.
} 
pessimisme très net devant le spectacle de 《la corruption du cœur humain ${ }^{17}$, une méfiance pour ce «tumulte des affaires » ${ }^{18}$ dans lequel évolue inévitablement l'homme public; de l'autre il opte pour un nécessaire engagement actif dans la cité, en faveur de «l'exercice de ces fonctions aussi glorieuses que pénibles » qui doivent susciter même une « sainte ambition ${ }^{19}$ ! Son pessimisme est fondé sur l'observation du monde : «On dit vrai si l'on ne veut parler que du fait... » Mais, s'empresse-t-il d'ajouter, «ce n'est pas par ce qui est, qu'il faut juger de ce qui doit être »; il faut donc soutenir «le Droit» contre «le Fait ${ }^{20}$, c'est-à-dire la «Raison ${ }^{21}$ contre la «folie» des hommes, la Vertu contre le Vice ${ }^{22}$. Aussi engage-t-il son fils à se préparer «aux grands emplois ». Contrairement au courant ultra janséniste, représenté par Barcos ou Singlin, par exemple ${ }^{23}$, il n'estime pas impossible au chrétien l'entrée en Politique. Au contraire, il n'hésite pas à fustiger ces «vertueux Solitaires [...] inutiles à leur Patrie $»^{24}$. Les termes ici peuvent-ils être innocents ? Retrouvant la veine et le souffle de Bossuet, d'Aguesseau voit en ceux « en qui réside la suprême Puissance », roi ou juges, «les images et les ministres de Dieu $»^{25}$. Fondé sur la Raison et sur la méthode des mathématiciens $(\text { sic })^{26}$, la théorie de d'Aguesseau est un véritable système politique, très spéculatif, certes, mais engagé. Sa pensée, par une oscillation subtile, reste cependant profondément marquée par Port-Royal :

«C'est en vain que je cherche en moi ces deux puissants secours [celui de la Connaissance, celui de la Force] ; la lumière et la force me manquent également dans le triste état où je suis réduit... J'entrevois le vrai bien et le vrai mal, jusqu'à un certain point ; mais ce vrai bien que j'aperçois et que j'aime naturellement, que je désire même de suivre, je ne le suis pas ; ce mal que je découvre aussi, que je déteste même, que je veux fuir véritablement, je ne le suis pas: presque toujours contraire à moi-même, approuvant ce que je ne fais point, et condamnant

\footnotetext{
${ }^{17}$ Instructions sur les études propres à former un magistrat, t. I, p. 327. Même expression dans Essai d'une institution au droit public, op. cit., t. I, p. 526.

${ }^{18}$ Ibid., p. 315.

${ }^{19}$ VIIIe Mercuriale. L'homme public..., op. cit., p. 123.

${ }^{20}$ Essai d'une institution au droit public, p. 526.

${ }^{21}$ Celle de Descartes, et déjà presque celle des Lumières.

${ }^{22}$ Essai d'une institution au droit public, p. 527.

${ }^{23}$ Cf. Isabelle Storez, Le chancelier Henri François d'Aguesseau (1668-1751), Publisud, Paris, 1996, p. 551 et s.

${ }^{24}$ Instruction sur les études propres..., op.cit., t. I, p. 287 (II ${ }^{\mathrm{e}}$ instruction. L'étude de l'histoire).

${ }^{25}$ Essai d'une institution au droit public, p. 539.

${ }^{26}$ Ibidem, p. 537. «Pour s'exprimer d'une manière géométrique », écrit encore d'Aguesseau (p. 543).
} 
ce que je fais, je trouve dans mon cœur et le coupable et le Juge, qui entretiennent une guerre continuelle; et malheureusement pour moi, c'est ordinairement le coupable qui est le plus fort, à la honte du Juge, réduit à déplorer le mal qu'il ne peut empêcher.

Serai-je donc surpris après cela d'entendre S. Paul même s'écrier : Malheureux que je suis, qui me délivrera de ce corps de mort? Et ma raison, si elle suit fidèlement ce qui résulte de la connoissance que j'ai de moi-même, ne me dictera-t-elle pas la réponse que le même Apôtre se fait en cet endroit ? Ce sera Dieu seul qui sera mon libérateur ; sa grâce seule peut et me montrer la route des véritables biens, et me donner les forces de la suivre $» .$.

On reconnaît des accents pascaliens. Peut-on attribuer au hasard cette dernière page de l'Essai d'une institution au Droit public ou considérer qu'elle révèle, entre le pessimisme janséniste et l'optimisme de la Raison, la boussole d'un équilibre intérieur? D'Aguesseau pose donc une condition à la justification de l'engagement politique : c'est qu' «il faut tout ramener à la Morale dans l'instruction des Grands » ${ }^{27}$. A l'inverse d'une sécularisation, dont il ne reniait pas tous les soubassements, en particulier gallicans, d'Aguesseau assure largement la légitimité du pouvoir, de l'autorité, et de l'exercice d'une « grande charge » sur la morale. Il se croyait certainement à la hauteur de cet idéal, malgré le temps, et les tempêtes (il l'a montré), mais pour autant ne surestimait-il guère ses qualités de politique. Cela nous ramène ainsi aux circonstances de son élévation.

\section{II - « Le procureur général fut bombardé chancelier »}

Le duc de Saint-Simon, dans un de ces raccourcis synthétiques dont il était orfèvre, rapporte ainsi l'élévation de d'Aguesseau à la chancellerie : «Le procureur général fut bombardé chancelier, mandé, trouvé à sa paroisse, et reçut les sceaux à l'instant que la famille de Voysin venoit de les apporter $»^{28}$. Dangeau affirme même que d'Aguesseau, ignorant la mort de Voysin, tomba des nues, ce que Saint-Simon corrige néanmoins : il raconte, en effet, qu'au cours d'un dîner, on vint annoncer à Saint-Contest, conseiller d'Etat et parent des d'Ormesson ${ }^{29}$, que le chancelier se mourait. Saint-Contest courut chez d'Aguesseau et «l'alla tirer le lendemain matin de son lit... pour lui apprendre cette subite et grande vacance, sur laquelle il est vrai que le procureur général

\footnotetext{
${ }^{27}$ Principe établi par Nicole auquel d'Aguesseau fait explicitement allusion. Cf. Isabelle Storez, op. cit., p. 565.

${ }^{28}$ Addition de Saint-Simon au Journal de Dangeau, op. cit., t. XVIII, p. 15.

${ }^{29}$ D'Aguesseau avait épousé en 1694 Anne Françoise Le Fèvre d'Ormesson.
} 
ne fit aucun mouvement, et s'en alla à sa grand'messe de paroisse. C'étoit le jour de la Chandeleur ${ }^{30}$. Le choix de d'Aguesseau s'opéra donc sans aucun doute dans un laps de temps très court, entre deux heures et huit ou neuf heures du matin, dans ce moment qui sépara le réveil du Régent de l'entretien qu'il eut avec d'Aguesseau, à qui l'on ne laissa d'ailleurs même pas le temps d'achever ses dévotions. Record! La personnalité de d'Aguesseau s'imposait-elle donc d'évidence?

En février 1717, Henri François d'Aguesseau avait exactement 48 ans, 2 mois et 5 jours $^{31}$. Un bel âge pour prétendre au ministère le plus prestigieux de la monarchie! Hormis Séguier en $1635^{32}$ et Pontchartrain en $1699^{33}$, nul chancelier depuis un siècle n' avait atteint cet honneur avant $63 \mathrm{ans}^{34}$. Le Régent recherchait spontanément la jeunesse : la dure mathématique de la démographie avait peuplé le Conseil de Régence de princes nés après 1670 et les membres les plus actifs du gouvernement, tels les ducs de Noailles ou de La Vrillière, n'avaient pas quarante ans.

Quant à sa carrière, qu'apportait-elle de si particulier que la réputation de d'Aguesseau s'imposât aussi aisément ? Entré en charge dès l'âge de 19 ans, après des études de droit complétées par les enseignements et l'expérience de son père, intendant et conseiller d'Etat, d'Aguesseau avait été successivement avocat général (1691-1700), puis procureur général du roi au Parlement de Paris. Carrière exclusivement parlementaire donc, mais au Parquet. En tant qu'avocat général, à l'audience, puis comme procureur général de façon plus administrative, d'Aguesseau avait constamment exercé une magistrature supérieure, intervenant dans les procès, pour défendre les intérêts du Roi, de la Loi, de l'Eglise, des mineurs et des communautés. Sa réputation se construisit alors sur son intense labeur, sur son intelligence et sur ses succès. "Il étoit l'aigle du Parlement » dit Saint-Simon ${ }^{35}$. Les contemporains furent sensibles à la réelle compétence de d'Aguesseau : les expressions hyperboliques surgissent

\footnotetext{
${ }^{30}$ Addition de Saint- Simon au Journal de Dangeau, op. cit., t. XVIII, p. 15.

${ }^{31}$ Il était né à Limoges le 27 novembre 1668.

${ }^{32}$ Pierre Séguier, né en 1588 , était dans sa quarante-huitième année.

33 A 56 ans, Pontchartrain entrait en charge beaucoup plus jeune que ses prédécesseurs, d'Aligre (82 ans !), Le Tellier (74 ans), et Boucherat (69 ans).

${ }^{34}$ De 1599 à 1717, la moyenne d'âge des chanceliers à leur nomination s'établit à plus de 65 ans

${ }^{35}$ Louis de Rouvroy, duc de Saint-Simon, Mémoires, éd. Boislisle, Paris, 1879-1930, t. III, p. 92.
} 
à tout moment sous leur plume, même des plus réticents. La marquise de Sévigné s'enthousiasmait pour «l'admirable avocat général»; l'avocat Barbier, Buvat, Narbonne, Luynes le créditent de toutes les vertus et de tous les talents, mais personne ne rend mieux que Saint-Simon l'estime dont on honorait le jeune magistrat au Parlement de Paris ${ }^{36}$. En bref, d'Aguesseau était de ces jeunes espoirs dont on pouvait attendre beaucoup et pour lesquels on pouvait solliciter jusqu' aux plus hautes fonctions. Lorsque fut vacante la place de premier président du Parlement de Paris, en avril 1707, par la démission d'Achille de Harlay, le bruit courut de la candidature de d'Aguesseau. Et SaintSimon d'y applaudir avec force, car il «n'avoit pour lui que cet appui de sa propre réputation qui, en tout genre, effaçoit toutes les autres du Parlement, et celle de son père, devant lequel toutes celles du Conseil disparoissoient...». L'échec de d'Aguesseau en cette rencontre n'empêche pas l'opinion de la Cour de lui rester favorable.

Il y avait donc une logique de compétence à placer à la tête de l'administration de la justice un magistrat brillant, qui avait su démontrer, outre ses compétences de jurisconsulte, ses incontestables talents d'administrateur ${ }^{37}$. L'originalité du choix venait plutôt de ce que Louis XIV avait préféré pour la chancellerie la robe des Conseils à celle des Parlements. Depuis le début du règne de Louis XIV, aucun chancelier n'avait effectué au Parlement, spécialement en celui de Paris, autre chose qu'un «stage» plus ou moins prolongé ; en revanche, quatre sur six avaient été un temps maîtres des requêtes de l'Hôtel ${ }^{38}$, trois étaient secrétaires et ministres d'Etat au moment de leur nomination ${ }^{39}$. Etendue aux gardes des sceaux qui permirent en cinq occasions d'écarter le chancelier du gouvernement, cette petite statistique est encore plus parlante : il avait fallu les périls de la Fronde pour que l'on osât choisir pour tenir les sceaux le premier président du Parlement de Paris, Mathieu Molé lui-

\footnotetext{
${ }^{36}$ Cf. la communication de Madame le Professeur Arlette Lebigre.

${ }^{37}$ Les attributions multiples du procureur général du roi faisaient entrer dans ses activités quotidiennes non seulement des tâches proprement judiciaires (conclusions dans les procès, audiences du parquet, jugement des conflits de juridictions, etc., correspondance avec les juridictions inférieures), mais aussi des attributions de police générale, avec en prime la tutelle des Universités, des Hôpitaux et des prisons. Un labeur écrasant dont d'Aguesseau surmonta les épreuves, même lors de la terrible crise de l'hiver 1709.

${ }^{38}$ Séguier, Le Tellier, Boucherat et Voysin.

${ }^{39}$ Le Tellier, Pontchartrain et Voysin.
} 
même, non sans tergiversations d'ailleurs ${ }^{40}$. Le Parlement, quant à lui, avait crié alors à la trahison ${ }^{41}$ ! Certes, tous, chanceliers et gardes des sceaux, appartenaient à la grande robe parisienne et leurs carrières avaient été savamment construites sur les jeux d'alliances avec le Parlement, les Pontchartrain avec les Talon ${ }^{42}$ et les Bignon, les Boucherat avec les Harlay, les Voysin avec les Lamoignon... Il n'en reste pas moins que le profil de carrière de d'Aguesseau contrastait avec ceux de ses prédécesseurs.

Qu'avait-il fallu de plus que sa réputation à d'Aguesseau pour atteindre, en ce temps record, une charge qu'il n'avait pas même postulée ? Un vide des candidatures ? Le coup de pouce d'un influent protecteur? Un échange de bons procédés ? Un petit tour d'horizon est nécessaire pour jauger le climat de cette nomination.

Dans l'ensemble, les sources contemporaines n'évoquent pas de concurrents à la chancellerie. Seul Buvat avance le nom d'un éventuel candidat, Amelot, que le Régent aurait même préféré, au fond, au procureur général. Michel-Jean Amelot, marquis de Gournay, renoue avec la tradition des grandes figures du Conseil de Louis XIV. Après un «apprentissage» de trois ans au Parlement de Paris, il était devenu maître des requêtes. Ambassadeur à Venise, au Portugal et en Suisse, il donna pleine satisfaction dans ces missions délicates. Conseiller d'Etat à partir de 1695 et directeur des affaires commerciales (1699), il révéla la plénitude de ses compétences lors de son ambassade extraordinaire à Madrid, à partir de 1705. Il avait réussi, écrit SaintSimon, «par beaucoup de logique, de sang-froid et d'impartialité ${ }^{43}$. Il rentra en 1709 précédé de la réputation d'avoir tenu l'Espagne à bout de bras pendant la pire période de la guerre. Comme le rapporte encore Saint-Simon, un vent de panique courut alors sur le ministère : "C'étoit, ce semble, le temps des orages à la cour: il en grondoit un qui menaçoit tous les ministres. Le retour d'Amelot... parut une bombe en l'air... Il y avoit été [en Espagne] à la tête de toutes les affaires, qu'il avoit trouvé dans le plus grand chaos et dans un épuisement étrange ; il gouverna les finances, le commerce, la marine, avec tant d'application et de succès que, malgré le malheur de la guerre, il les rétablit

\footnotetext{
${ }^{40}$ Appelé le 5 avril 1651, Molé perdit les sceaux le 13 du même mois, pour les reprendre le 9 septembre suivant (jusqu'à sa mort le 3 janvier 1656).

${ }^{41} C f$. Jean Le Boindre, Débats du Parlement pendant la Minorité de Louis XIV, t. II, textes établis et présentés par Isabelle Storez-Brancourt, éd. Honoré Champion, Paris, 2002.

${ }^{42}$ Comme d'Aguesseau.

${ }^{43}$ Saint-Simon, Mémoires, op. cit., t. IV, p. 285.
} 
dans le plus grand ordre, les augmenta considérablement, acquitta une infinité de choses, régla les troupes... Cela parut une création ${ }^{44}$. En février 1717 , Amelot venait de fêter ses 62 ans. A tout point de vue, il offrait le profil même de ceux qui retenaient l'attention de Louis XIV, mais, avec la Régence, les temps avaient changé.

En fait, si en 1709 Amelot n'avait pas connu l'élévation que l'on attendait, c'est que « le soupçon de jansénisme, si aisé à donner et à prendre », selon le mot de Saint-Simon, frappait le marquis de Gournay tout autant que les d'Aguesseau. Ils étaient d'ailleurs les meilleurs amis du monde et tandis que le père faisait transmettre à Amelot le plan d'étude qu'il avait composé pour ses enfants ${ }^{45}$, le fils confiait l'éducation de sa fille à la Mère Amelot de Gournay, au couvent de la Visitation-Sainte-Marie. Amelot reprit donc sa place comme conseiller d'Etat et, jusqu'en 1716, y collabora avec son ami Henri d'Aguesseau. Il y avait dans cette teinture de jansénisme le seul point commun entre le procureur général et le conseiller d'Etat. En 1717, c'était plutôt porteur.

Entre Amelot et d'Aguesseau, dans le plateau de la balance, il y eut en fait, pour d'Aguesseau, l'influence de Noailles. Adrien Maurice, duc de Noailles (1678-1766), avait accompagné Philippe V en Espagne, en 1705, et participé aux opérations les plus importantes de la guerre de Succession d'Espagne. En 1715, il se présentait à Philippe d'Orléans dans la pleine forme de ses ambitions et de sa maturité. Il occupait la place clef de président du Conseil des Finances ${ }^{46}$. Jean Buvat détaille ainsi la scène ${ }^{47}$ de son intervention auprès du Régent : "Peu après, M. le duc de Noailles étant arrivé au Palais Royal, dit à ce prince: Votre Altesse Royale peut brusquer cette affaire et se déterminer au plus tôt sur le sujet qu'elle juge digne de remplir la charge de chancelier de France, pour ne pas être accablé de sollicitations. Sur quoi, le

\footnotetext{
${ }^{44}$ Ibidem, p. 81-82.

${ }^{45}$ C'est le titre-même du manuscrit de ce Plan d'étude qui nous en garantit le destinataire (BnF, Nouvelles acquisitions françaises, 1991).

46 Dans le système de la polysynodie que le Régent avait repris des projets du duc de Bourgogne et des grands seigneurs réactionnaires de son entourage, un tel poste représentait une puissance ministérielle considérable. Saint-Simon affirme, malgré son hostilité personnelle à Noailles, qu'il encouragea le Régent dans ce choix : «Je le [Noailles] croyoit encore capable de travail par lui-même, instruit sur tout comme il l'étoit depuis deux ans par Desmaretz. Ses richesses et ses établissements m'assuroient de la netteté de ses mains ; son ambition même, de tous les efforts à bien faire dans une place si considérable...»(Mémoires, op. cit., t. XXIX, p. 54).

${ }^{47}$ Jean Buvat, Journal de la Régence, éd. Campardon, Paris, 1865, t. I, p. 245.
} 
prince dit aussitôt à un gentilhomme d'aller chez M. d'Aguesseau, procureur général ». Saint-Simon, pour sa part, rapporte les faits de façon plus cocasse : en ce matin du 2 février, le Régent était à sa toilette lorsqu'arriva le duc de Noailles : ce dernier «en profita et tira si bien sur le temps, que sur cette chaise percée, le procureur général fut bombardé chancelier ${ }^{48}$. Saint-Simon revient plusieurs fois sur l'intervention de Noailles dans cette circonstance : «Il étoit visible qu'il avoit fait le chancelier», raille-t-il, «et il étoit bien aise que personne n'en doutât ». D'ailleurs le maréchal de Villars n'en doutait pas et dit que la place fut donnée à d'Aguesseau « fort lié avec le duc de Noailles ${ }^{49}$.

L'amitié des d'Aguesseau pour les Noailles est un fait avéré et déjà ancien en $1717^{50}$. Elle se prolongea bien au-delà de cette date. Les affaires religieuses, spécialement le jansénisme dont on taxait le cardinal de Noailles autant que les d'Aguesseau, l'hostilité commune, et tout à fait réelle, aux jésuites et à leur influence auprès des rois, avait cimenté des liens de confiance et d'estime réciproques. En contrepartie, on apprend par Saint-Simon les craintes qu'inspiraient au duc de Noailles les trop évidentes compétences d'Amelot de Gournay en matière de finances: Amelot, qui rentrait d'une mission à Rome au lendemain de la mort de Louis XIV, fut bien reçu mais, écrit finement Saint-Simon, «sa réputation trop justement établie blessa la jalousie du maréchal d'Huxelles... Elle n'inquiéta pas moins Noailles et Rouillé... Il ne put donc entrer dans le Conseil des affaires étrangères, ni dans celui des finances, lui qui auroit été si utilement et si convenablement placé dans celui de régence, et jamais il ne fut consulté sur rien ${ }^{51}$. Aussi ne fut-il pas difficile à Noailles de souligner au Régent tous les avantages que présentait d'Aguesseau sur Amelot : sa compétence et son expérience de l'administration de la justice en un temps où l'on ressentait fortement la nécessité d'une réforme des offices de judicature, des procédures et du corps même des lois; sa fermeté de caractère, qu'il avait révélée lors du conflit entre le Parlement de Paris et Louis XIV à propos d'Unigenitus; son absence d'ambition et sa modestie universellement appréciées... Le Régent d'ailleurs redoutait Amelot «par l'union avec laquelle il avoit vécu avec la princesse des Ursins en Espagne, où

\footnotetext{
${ }^{48}$ Saint-Simon, Addition au Journal de Dangeau, op. cit., t. XVIII, p. 15.

${ }^{49}$ Villars, Mémoires, Société de l'Histoire de France, Paris, 1904, t. IV, p. 93.

50 Les premiers contacts avaient été pris lorsqu'Henri d'Aguesseau était intendant du Languedoc.

${ }^{51}$ Saint-Simon, Mémoires, op. cit., t. XXIX, p. 134-135.
} 
sous le nom d'ambassadeur il avoit fait la fonction de premier ministre $»^{52}$. On voit que la compétition ne fut pas bien rude. Noailles l'enleva facilement.

Philippe d'Orléans se souvenait-il aussi que le procureur général avait été des principaux magistrats impliqués dans la modification du testament de Louis XIV dont, le 26 août 1714, il avait reçu, en présence du premier président de Mesmes, le dépôt en mains propres ? Les dispositions, dont le sens global avait filtré bien avant la mort du roi, étaient inacceptables pour le Régent dont le pouvoir se trouvait strictement délimité dans une structure, en revanche, favorable aux bâtards royaux ainsi qu'à la permanence du personnel politique de la fin du règne. Le $1^{\text {er }}$ septembre 1715 , «le duc d'Orléans », affirme Narbonne, «courut à Paris et mit de son parti le procureur général ${ }^{53}$. Ce fut ainsi sur la requête expresse de d'Aguesseau que le Parlement leva toutes les entraves que le testament imposait à l'autorité du Régent. «M. Daguesseau », rapporte également le maréchal de Villars, «proposa de la part du duc d'Orléans, plusieurs conseils ». Le maréchal de Villars «présenta avec force que dans les premiers moments d'une nouvelle administration il ne fallait pas renverser tout l'ordre anciennement établi... Le procureur général répondit que M. le duc d'Orléans étoit entièrement déterminé à ces nouveaux conseils $»^{54}$. D'Aguesseau gagna à cette attitude, d'abord, une place au Conseil des Affaires ecclésiastiques, aux côtés du cardinal de Noailles. Il n'est pas impossible que le Régent se soit encore souvenu avec reconnaissance de ces événements capitaux du 2 septembre 1715 au petit matin de ce 2 février 1717.

La faiblesse des intrigues qui soutinrent la nomination d'Henri François d'Aguesseau à la Chancellerie de France contraste avec la quasi unanimité des applaudissements qui accompagnèrent son élévation : «la nouvelle ayant été répandue dans tout Paris », écrit Buvat, «ce choix a été universellement approuvé $»^{55}$. On se presse pour féliciter. Le nouveau chancelier, quant à lui, était éberlué : "Daguesseau, dans sa surprise», écrit Saint-Simon, "ne vit qu'un étang » 56 !

\footnotetext{
${ }^{52}$ Ibidem, t. XXIX, p. 134.

${ }^{53}$ Pierre Narbonne, Journal des règnes de Louis XIV et Louis XV de l'année 1701 à 1744, Paris, 1866, in $-8^{\circ}$, p. 152.

${ }^{54}$ Villars, op. cit., t. IV, p. 73.

55 Jean Buvat, Gazette de la Régence, éd. Barthélemy, Paris, 1887, p. 145.

${ }^{56}$ Saint-Simon, Mémoires, op. cit., t. XXXI, p. 23.
} 
Le choix de d'Aguesseau, c'était l'apothéose de la rentrée triomphale que le Parlement de Paris avait faite, en septembre 1715, dans la politique : le promoteur même de la restitution du droit de remontrance préalable à l'enregistrement des ordonnances était porté au pinacle : le rôle personnel de d'Aguesseau dans la négociation avec le Régent, sur cette question essentielle de la place du Parlement dans la structure monarchique, bien qu'occulte, apparaît clairement, non seulement dans le texte de la mercuriale de la SaintMartin $1715^{57}$, mais aussi dans son ouvrage inachevé, très important, intitulé Fragmens sur l'origine et l'usage des remontrances.

D'Aguesseau était aussi le candidat rêvé du parti janséniste qui, pour l'heure, se croyait le vent en poupe : «Vous concevez, par cet incident », écrit Jean Buvat, «que la Constitution et les jésuites perdent un appui considérable... tandis que l'autre parti gagne une personne ferme, habile et zélée pour l'Eglise gallicane ${ }^{58}$. «Je sais », affirme même cet auteur, «qu'à cause de la nomination du nouveau chancelier les jésuites sont dans la dernière consternation $»^{59}$.

Il y avait là, apparemment, tous les ingrédients de la «réaction », de cette flambée de contradictions au règne de Louis XIV, dans les aspects qui avaient le plus fortement marqué ses dernières années, c'est-à-dire, l'hostilité aux jansénistes et le domptage du Parlement. Peut-être, au fond, y avait-il quelque ambiguïté à prendre d'Aguesseau pour un soutien à la réaction politique qui s'exprimait principalement par la voix des grands seigneurs ? L'analyse de sa pensée politique n'indique aucune des composantes de la réaction aristocratique : ni le fervent appel aux Etats généraux (Saint-Simon, Boulainvilliers), ni la restitution du pouvoir à l'Epée au détriment de la Plume, ni même la critique de la centralisation opérée, dans le ministère, au profit du Conseil secret et, dans les provinces, au profit des intendants. D'Aguesseau est, au rôle du Parlement près, un partisan de la monarchie « absolue », et surtout un partisan de l'Etat. Les Noailles et autres grands seigneurs n'en étaient pas à une analyse aussi fine ! Les apparences lui étaient favorables, tandis qu'Amelot, que l'on taxait aussi de janséniste, il est vrai, entrait manifestement mal dans le cadre de la polysynodie, et encore plus mal dans l'éventualité d'une politique de rapprochement avec l'Angleterre qui contrarierait l'alliance louis-

\footnotetext{
${ }^{57}$ Euvres du chancelier d'Aguesseau, t. I, p. 235.

${ }^{58}$ Jean Buvat, Gazette de la Régence, p. 145.

${ }^{59}$ Ibidem, p. 146.
} 
quatorzienne avec l'Espagne Bourbon. «C'était [pourtant] », regrette SaintSimon à propos d'Amelot, «dans ces temps de désastres, le comble de la capacité, et en même temps celui de l'esprit, de l'adresse et du liant ${ }^{60}$. Il eût fallu au Régent une réelle ligne politique à long terme. Sur l'instant, tout semblait faire de d'Aguesseau le chancelier idéal.

«Tenez, Monsieur d'Aguesseau, gardez les clefs de la chancellerie jusqu'à ce que je vous les redemande », aurait dit en riant le duc d'Orléans au procureur général ; «Tenez encore une fois, prenez-les et ne faites pas tant le benêt. Il suffit que je connaisse ce que vous savez faire ${ }^{61}$. Moins d'un an plus tard, puis encore une fois en février 1722, le Régent devait démontrer que ce «jusqu'à ce que je vous les redemande»n'était pas une vaine formule. «Je n'ai mérité ni l'honneur de recevoir les sceaux, ni l'affront d'en être privé », commenta sobrement d'Aguesseau ${ }^{62}$. Ces «hoquets ${ }^{63} \mathrm{du}$ chancelier dans l'exercice de ses fonctions, qui entraînèrent deux puis cinq ans d'exil à Fresnes, révèlent plus que tout la fragilité des bases politiques du choix opéré le 2 février 1717. Si la disgrâce de 1722 apparaît liée à l'ambition de Dubois, donc à une affaire de personne, les événements de janvier 1718 relient directement l'échec politique de d'Aguesseau lors de la montée de l'influence de Law aux ingrédients de l'intrigue de février 1717. D'Aguesseau représentait le parti du travail, de l'intégrité, de l'austérité et de la fidélité : il était appelé à collaborer avec un duc d'Orléans vif au jeu politique, léger et versatile. L'un concevait la chancellerie comme une mission de salut public, l'autre comme «un siège éjectable». Quel qui pro quo! En 1718, le Régent ne voulait plus entendre parler ni des jansénistes, ni du Parlement qui semait sa route d'embûches, ni des finances de Noailles. Il voulait Law, la rapidité de l'action politique et une grande souplesse d'échine de la part de son chancelier. Trop attaché à Noailles, d'Aguesseau sombra dans la nouvelle mue de la monarchie. Et l'on chansonna sur le Pont-Neuf :

« Je ne regrette pas Noailles Car il ne faisait rien qui vaille ;

Pour Daguesseau il étoit bon Charitable, aimant la justice, Mais qui veut imiter Néron

\footnotetext{
${ }^{60}$ Saint-Simon, Mémoires, t. XXIX, p. 134.

${ }^{61}$ Jean Buvat, Journal de la Régence, p. 148.

${ }^{62}$ Louis-Philippe, comte de Ségur, Notice sur le chancelier d'Aguesseau, Paris, 1822, p. 15.

${ }^{63}$ Expression de Mathieu Marais.
} 


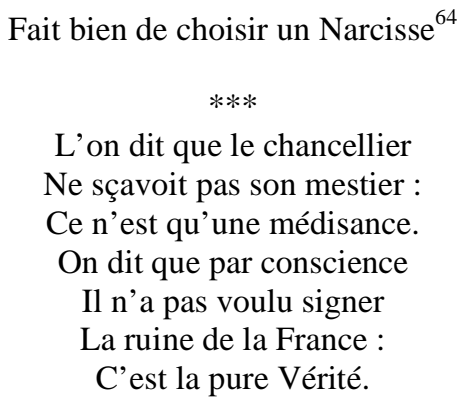

Lorsqu'en 1720, d'Aguesseau, rappelé en hâte, se fit le liquidateur de l'expérience de Law, on l'accusa de bassesse : Et homo factus est ${ }^{65}$ ! Lorsque la même année, il obtint du Parlement l'enregistrement forcé d'Unigenitus, on dénonça son inutile «félonnie ${ }^{66}$. D'Aguesseau y perdit à jamais sa réputation de «politique » et Saint-Simon foudroie ainsi le malheureux ministre : «Avec un des plus beaux et lumineux esprits de son siècle... jamais rien de si hermétiquement bouché en fait de finances, d'affaires d'Etat, de connoissance du monde, ni de si incapable d'y rien apprendre $»^{67}$. Sans appel !

A long terme, cependant, le «mauvais choix » du Régent en 1717 se révèle, comme par anticipation, l'option par excellence du roi Louis $\mathrm{XV}$ : d'Aguesseau dont on décriait l'inaptitude aux combinaisons politiciennes ${ }^{68}$ répondait à l'horreur que nourrissait ce roi pour les intrigues en coulisses. L'un et l'autre en furent sans doute des victimes de choix. Moraliste et janséniste dans les profondeurs de sa philosophie politique, d'Aguesseau, au ministère, rejeta «une politique janséniste » qui eût été à ses yeux aussi partisane que subversive. Le roi et son chancelier avait un goût commun pour le travail de cabinet, pour le savoir, pour une certaine idée de la justice, pour la stabilité : cette concordance fut la force de d'Aguesseau à la chancellerie où il remporta, pour le XVIII ${ }^{\mathrm{e}}$ siècle, la palme de la longévité ministérielle.

\footnotetext{
${ }^{64}$ Le comte d'Argenson devenu garde des sceaux. Bibl. Nat., Nouv. acq. fr. 2483, f ${ }^{\circ} 148$.

${ }^{65}$ Placard apposé sur la porte de la chancellerie.

${ }^{66}$ Bibl. Nat., fonds Joly de Fleury, ms. fr. 25570 , f 528.

${ }^{67}$ Saint-Simon, Mémoires, t. XXXIII, p. 43.

${ }^{68}$ «Cet esprit doux, incertain, qui se trouveroit comme un aveugle au milieu du bruit et des cabales », écrit Saint-Simon (Mémoires, t. XXXI, p. 21). « Son louche et son gauche en matière d'Etat le déprisa beaucoup » (ibidem, t. XXXIII, p. 6).
} 